\title{
New 2D parallel thinning algorithms based on critical kernels
}

\author{
Gilles Bertrand and Michel Couprie \\ Institut Gaspard-Monge \\ Laboratoire A2SI, Groupe ESIEE \\ Cité Descartes, BP 99 \\ 93162 Noisy-le-Grand Cedex France \\ g.bertrand@esiee.fr,m.couprie@esiee.fr
}

\begin{abstract}
Critical kernels constitute a general framework settled in the category of abstract complexes for the study of parallel thinning in any dimension. In this context, we propose several new parallel algorithms, which are both fast and simple to implement, to obtain symmetrical skeletons of $2 \mathrm{D}$ objects in 2D or 3D grids. We prove some properties of these skeletons, related to topology preservation, and to the inclusion of the topological axis which may be seen as a generalization of the medial axis.
\end{abstract}

\section{Introduction}

Forty years ago, in 1966, D. Rutovitz proposed an algorithm which is certainly the first parallel thinning algorithm [23]. Since then, many 2D parallel thinning algorithms have been proposed, see for example $[25,1,19,7,11,13,9,18]$. A fundamental property required for such algorithms is that they do preserve the topology of the original objects. In fact, such a guarantee is not obvious to obtain, even for the 2D case, see [8] where some counter-examples are given.

In [3], one of the authors introduces a general framework for the study of parallel thinning in any dimension in the context of abstract complexes. A new definition of a simple point (a point which may be deleted without "changing the topology of the object") has been proposed, this definition is based on the collapse operation which is a classical tool in algebraic topology and which guarantees topology preservation. The most fundamental result proved in [3] is that, if a subset $Y$ of $X$ contains the critical kernel of $X$, then $Y$ has the same topology as $X$.

In this paper, we focus on 2D structures in 2D and 3D spaces. We introduce the notions of crucial faces and pixels (Sec. 4, Sec. 5) which permit to make a link with the framework of digital topology [16]. Thanks to simple local characterizations, we are able to express thinning algorithms by the way of sets of masks, as in most papers related to parallel thinning. We introduce the formal definition of a minimal symmetric skeleton, and we propose an algorithm to compute it (Sec. 6). The quality of a curvilinear skeleton is often assessed by the fact that it contains, approximately or completely, the medial axis of the shape. We introduce the topological axis (Sec. 7), a generalization of the medial axis (which is 
not defined for the case of two-dimensional structures in discrete $n$-dimensional spaces, $n>2$ ). In $2 \mathrm{D}$, we propose a new parallel algorithm (Sec. 8) to compute skeletons which are guaranteed to include the medial axis. We extend our algorithms to the $3 \mathrm{D}$ case by proposing a new algorithm to compute minimal symmetric skeletons of $2 \mathrm{D}$ objects in $3 \mathrm{D}$ grids, and also a new algorithm to compute skeletons of $2 \mathrm{D}$ objects in $3 \mathrm{D}$ grids which are guaranteed to contain the topological axis (Sec. 9).

For the sake of space, proofs are not given in this paper, most of them may be found in [3] or [5].

\section{Cubical complexes}

In this section, we give some basic definitions for cubical complexes, see also [17]. We consider here only the two-dimensional case. The reader is invited to check that many of the notions introduced in the first sections make sense in arbitrary $n$-dimensional cubical spaces.

If $T$ is a subset of $S$, we write $T \subseteq S$, we also write $T \subset S$ if $T \subseteq S$ and $T \neq S$. Let $\mathbb{Z}$ be the set of integers. We consider the families of sets $\mathbb{F}_{0}^{1}, \mathbb{F}_{1}^{1}$, such that $\mathbb{F}_{0}^{1}=\{\{a\} \mid a \in \mathbb{Z}\}, \mathbb{F}_{1}^{1}=\{\{a, a+1\} \mid a \in \mathbb{Z}\}$. A subset $f$ of $\mathbb{Z}^{n}, n \geq 2$, which is the Cartesian product of exactly $m$ elements of $\mathbb{F}_{1}^{1}$ and $(n-m)$ elements of $\mathbb{F}_{0}^{1}$ is called a face or an $m$-face of $\mathbb{Z}^{n}, m$ is the dimension of $f$, we write $\operatorname{dim}(f)=m$. We denote by $\mathbb{F}_{2}^{n}$ the set composed of all $m$-faces of $\mathbb{Z}^{n}, m=0,1,2$ and $n \geq 2$. An $m$-face of $\mathbb{Z}^{n}$ is called a point if $m=0$, a (unit) interval if $m=1$, a (unit) square if $m=2$.

In this paper, we will consider only $2 \mathrm{D}$ objects which are in $2 \mathrm{D}$ or $3 \mathrm{D}$ spaces. Thus, in the following, we suppose that $n=2$ or $n=3$.

Let $f$ be a face in $\mathbb{F}_{2}^{n}$. We set $\hat{f}=\left\{g \in \mathbb{F}_{2}^{n} \mid g \subseteq f\right\}$ and $\hat{f}^{*}=\hat{f} \backslash\{f\}$. Any $g \in \hat{f}$ is a face of $f$, and any $g \in \hat{f}^{*}$ is a proper face of $f$.

If $X$ is a finite set of faces in $\mathbb{F}_{2}^{n}$, we write $X^{-}=\cup\{\hat{f} \mid f \in X\}, X^{-}$is the closure of $X$.

A set $X$ of faces in $\mathbb{F}_{2}^{n}$ is a cell or an $m$-cell if there exists an $m$-face $f \in X$, such that $X=\hat{f}$. The boundary of a cell $\hat{f}$ is the set $\hat{f}^{*}$.

A finite set $X$ of faces in $\mathbb{F}_{2}^{n}$ is a complex $\left(\right.$ in $\mathbb{F}_{2}^{n}$ ) if $X=X^{-}$. Any subset $Y$ of a complex $X$ which is also a complex is a subcomplex of $X$. If $Y$ is a subcomplex of $X$, we write $Y \preceq X$. If $X$ is a complex in $\mathbb{F}_{2}^{n}$, we also write $X \preceq \mathbb{F}_{2}^{n}$.

Let $X \preceq \mathbb{F}_{2}^{n}$. A face $f \in X$ is principal for $X$ if there is no $g \in X$ such that $f \in \hat{g}^{*}$. We denote by $X^{+}$the set composed of all principal faces of $X$. Observe that, in general, $X^{+}$is not a complex, and that $\left[X^{+}\right]^{-}=X$.

Let $X \preceq \mathbb{F}_{2}^{n}, \operatorname{dim}(X)=\max \left\{\operatorname{dim}(f) \mid f \in X^{+}\right\}$is the dimension of $X$. We say that $X$ is an $m$-complex if $\operatorname{dim}(X)=m$.

We say that $X$ is pure if, for each $f \in X^{+}$, we have $\operatorname{dim}(f)=\operatorname{dim}(X)$.

Let $X \preceq \mathbb{F}_{2}^{n}$ and $Y \preceq X$. If $Y^{+} \subseteq X^{+}$, we say that $Y$ is a principal subcomplex of $X$ and we write $Y \sqsubseteq X$. Observe that, for any $X \preceq \mathbb{F}_{2}^{n}, \emptyset \sqsubseteq X$.

If $X \preceq \mathbb{F}_{2}^{n}$ and if $X$ is a pure 2-complex, we also write $X \sqsubseteq \mathbb{F}_{2}^{n}$. 
Let $X \preceq \mathbb{F}_{2}^{n}$ and let $Y \preceq X$. We set $X \otimes Y=\left[X^{+} \backslash Y^{+}\right]^{-}$. The set $X \otimes Y$ is a complex which is the detachment of $Y$ from $X$.

Two distinct faces $f$ and $g$ of $\mathbb{F}_{2}^{n}$ are adjacent if $f \cap g \neq \emptyset$. Two complexes $X, Y$ in $\mathbb{F}_{2}^{n}$ are adjacent if there exist $f \in X$ and $g \in Y$ which are adjacent.

Let $X \preceq \mathbb{F}_{2}^{n}$. A sequence $\pi=\left\langle f_{0}, \ldots, f_{l}\right\rangle$ of faces in $X$ is a path in $X$ (from $f_{0}$ to $f_{l}$ ) if $f_{i}$ and $f_{i+1}$ are adjacent for each $i=0, \ldots, l-1$; the number $l$ is the length of $\pi$. We say that $X$ is connected if, for any pair of faces $(f, g)$ in $X$, there is a path in $X$ from $f$ to $g$. We say that $Y \preceq X$ is a connected component of $X$ if $Y \subseteq X, Y$ is connected, and if $Y$ is maximal for these two properties (i.e., we have $Z=Y$ whenever $Y \preceq Z \preceq X$ and $Z$ connected).

Two 2-faces $f$ and $g$ of $\mathbb{F}_{2}^{n}$ are strongly adjacent if $f \cap g$ is a 1-face.

Let $X \sqsubseteq \mathbb{F}_{2}^{n}$. A sequence $\pi=\left\langle f_{0}, \ldots, f_{l}\right\rangle$ of 2 -faces in $X$ is a strong path in $X$ (from $f_{0}$ to $f_{l}$ ) if $f_{i}$ and $f_{i+1}$ are strongly adjacent for each $i=0, \ldots, l-1$; the number $l$ is the length of $\pi$. We say that $X$ is strongly connected if, for any pair of 2 -faces $(f, g)$ in $X$, there is a strong path in $X$ from $f$ to $g$.

If $f$ is a 2 -face of $\mathbb{F}_{2}^{n}$, we set:

$\Gamma^{*}(f)=\left\{g \in \mathbb{F}_{2}^{n} \mid g\right.$ is a 2-face adjacent to $\left.f\right\}, \Gamma(f)=\Gamma^{*}(f) \cup\{f\}$; and

$\Gamma_{\mathcal{S}}^{*}(f)=\left\{g \in \mathbb{F}_{2}^{n} \mid g\right.$ is strongly adjacent to $\left.f\right\}, \Gamma_{\mathcal{S}}(f)=\Gamma_{\mathcal{S}}^{*}(f) \cup\{f\}$.

\section{Simple cells}

Intuitively a cell $\hat{f}$ of a complex $X$ is simple if its removal from $X$ "does not change the topology of $X$ ". In this section we propose a definition of a simple cell based on the operation of collapse [10], which is a discrete analogue of a continuous deformation (a homotopy). Note that this definition is a rather general one, in particular, it may be directly extended to $n$-dimensional cubical complexes [3].

Let $X$ be a complex in $\mathbb{F}_{2}^{n}$ and let $f \in X^{+}$. The face $f$ is a border face for $X$ if there exists one face $g \in \hat{f}^{*}$ such that $f$ is the only face of $X$ which contains $g$. Such a face $g$ is said to be free for $X$ and the pair $(f, g)$ is said to be a free pair for $X$. We say that $f \in X^{+}$is an interior face for $X$ if $f$ is not a border face. In Fig. $1(\mathrm{a})$, the pair $(f, j)$ is a free pair for $X$, and the complex $X$ has no interior face.

Let $X$ be a complex, and let $(f, g)$ be a free pair for $X$. The complex $X \backslash\{f, g\}$ is an elementary collapse of $X$.

Let $X, Y$ be two complexes. We say that $X$ collapses onto $Y$ if there exists a collapse sequence from $X$ to $Y$, i.e., a sequence of complexes $\left\langle X_{0}, \ldots, X_{l}\right\rangle$ such that $X_{0}=X, X_{l}=Y$, and $X_{i}$ is an elementary collapse of $X_{i-1}, i=1, \ldots, l$; the number $l$ is the length of the collapse sequence. If $X$ collapses onto $Y$, we also say that $Y$ is a retraction of $X$. See illustration Fig. 1 (a), (b), (c).

We give now a definition of a simple point, it may be seen as a discrete analogue of the one given by T.Y. Kong in [15] which lies on continuous deformations in the $n$-dimensional Euclidean space.

Definition 1. Let $X \preceq \mathbb{F}_{2}^{n}$. Let $f \in X^{+}$.

We say that $\hat{f}$ and $f$ are simple for $X$ if $X$ collapses onto $X \otimes \hat{f}$. 
(a)
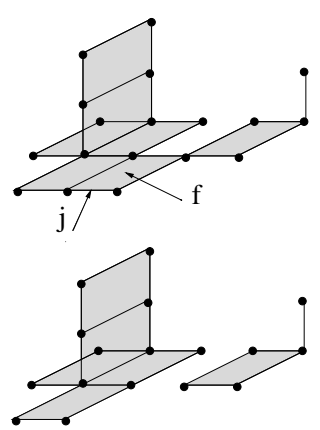

(b)

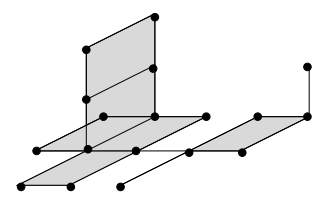

b)

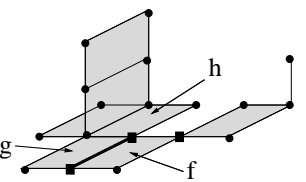

(c)
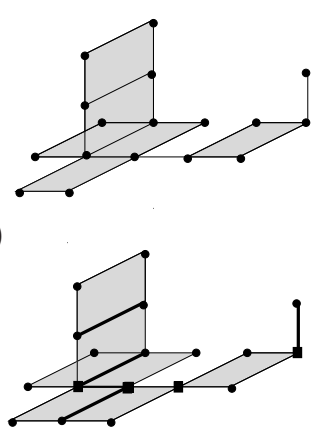

(d)

(e)

(f)

Fig. 1. (a) A complex $X$, (b) and (c) two steps of elementary collapse of $X$, (d) the detachment of $\hat{f}$ from $X$, (e) the attachment of the 2 -face $f$ is highlighted, the face $f$ is not simple, whereas $g$ and $h$ are simple, (f) the essential 0 - and 1-faces for $X$ are highlighted.

The notion of attachment, as introduced by T.Y. Kong [14, 15], leads to a local characterization of simple cells.

Definition 2. Let $X \preceq \mathbb{F}_{2}^{n}$ and let $f \in X^{+}$. The attachment of $\hat{f}$ for $X$ is the complex $\operatorname{Attach}(\hat{f}, X)=\hat{f}^{*} \cap[X \odot \hat{f}]$.

In other words, a face $g$ is in $\operatorname{Attach}(\hat{f}, X)$ if $g$ is in $\hat{f}^{*}$ and if $g$ is a (proper) face of a principal face $h$ distinct from $f$.

The following proposition is an easy consequence of the above definitions.

Proposition 3. Let $X \preceq \mathbb{F}_{2}^{n}$, and let $f \in X^{+}$.

The cell $\hat{f}$ is simple for $X$ if and only if $\hat{f}$ collapses onto $\operatorname{Attach}(\hat{f}, X)$.

The attachment of a 2-face $f$ of a complex $X$ is highlighted Fig. 1 (e) and $X \otimes \hat{f}$ is depicted in (d). It may be seen that $f$ is not simple: there is no collapse sequence from $X$ (a) to $X \ominus \hat{f}(\mathrm{~d})$. On the other hand the faces $g$ and $h$ are simple. The next property may be directly derived from Prop. 3.

Proposition 4. Let $X \preceq \mathbb{F}_{2}^{n}$, and let $f \in X^{+}$.

1) If $\hat{f}$ is a 0 -cell, then $\hat{\hat{f}}$ is not simple for $X$;

2) If $\hat{f}$ is a 1-cell, then $\hat{f}$ is simple for $X$ if and only if Attach $(\hat{f}, X)$ is made of a single point;

3) If $\hat{f}$ is a 2-cell, then $\hat{f}$ is simple for $X$ if and only if $f$ is a border face and $\operatorname{Attach}(\hat{f}, X)$ is non-empty and connected.

From Prop. 4, we easily derive a characterization of simple 2-faces which is an equivalent, in the framework of $2 \mathrm{D}$ complexes in $\mathbb{F}_{2}^{n}$, of the well-known characterization of simple pixels in the square grid given by A. Rosenfeld [21].

Proposition 5. Let $X \sqsubseteq \mathbb{F}_{2}^{n}$, and let $f$ be a 2-face for $X$. The face $f$ is simple for $X$ if and only if:

i) $f$ is a border face; and

ii) $\Gamma^{*}(f) \cap X$ is non-empty and connected. 


\section{Critical kernels}

Let $X$ be a complex in $\mathbb{F}_{2}^{n}$. We observe that, if we remove simultaneously simple cells from $X$, we may obtain a set $Y$ such that $X$ does not collapse onto $Y$. In other words, if we remove simple cells in parallel, we may "change the topology" of the original object $X$. For example, in Fig. 1 (e), $g$ and $h$ are simple for $X$, but the complexes $X$ and $X \odot[\hat{g} \cup \hat{h}]$ have not "the same topology" (here, the same number of connected components). Thus, it is not possible to use directly the notion of simple cell for thinning discrete objects in a symmetrical manner. In this section, we introduce a new framework for thinning in parallel discrete objects with the warranty that we do not alter the topology of these objects. This method may be extended for complexes of arbitrary dimension [3]. As far as we know, this is the first method which allows to thin arbitrary complexes in a symmetric way.

This method is based solely on three notions, the notion of an essential face which allows to define the core of a face, and the notion of a critical face.

Definition 6. Let $X \preceq \mathbb{F}_{2}^{n}$ and let $f \in X$. We say that $f$ is an essential face for $X$ if $f$ is precisely the intersection of all principal faces of $X$ which contain $f$, i.e., if $f=\cap\left\{g \in X^{+} \mid f \subseteq g\right\}$. We denote by $E s s(X)$ the set composed of all essential faces of $X$. If $f$ is an essential face for $X$, we say that $\hat{f}$ is an essential cell for $X$.

Observe that a principal face for $X$ is necessarily an essential face for $X$, i.e., $X^{+} \subseteq \operatorname{Ess}(X)$. The essential 0- and 1-faces of the complex $X$ of Fig. 1 (a) are highlighted Fig. 1 (f).

Definition 7. Let $X \preceq \mathbb{F}_{2}^{n}$ and let $f \in \operatorname{Ess}(X)$. The core of $\hat{f}$ for $X$ is the complex, denoted by $\operatorname{Core}(\hat{f}, X)$, which is the union of all essential cells for $X$ which are in $\hat{f}^{*}$, i.e., Core $(\hat{f}, X)=\cup\left\{\hat{g} \mid g \in \operatorname{Ess}(X) \cap \hat{f}^{*}\right\}$.

The preceding definition may be seen as a generalization of the notion of attachment for arbitrary essential cells (not necessarily principal).

Proposition 8. Let $X \preceq \mathbb{F}_{2}^{n}$ and let $f \in X^{+}$. The attachment of $\hat{f}$ for $X$ is precisely the core of $\hat{f}$ for $X$, i.e, we have $\operatorname{Attach}(\hat{f}, X)=\operatorname{Core}(\hat{f}, X)$.

Definition 9. Let $X \preceq \mathbb{F}_{2}^{n}$ and let $f \in X$. We say that $f$ and $\hat{f}$ are regular for $X$ if $f \in \operatorname{Ess}(X)$ and if $\hat{f}$ collapses onto $\operatorname{Core}(\hat{f}, X)$. We say that $f$ and $\hat{f}$ are critical for $X$ if $f \in E s s(X)$ and if $f$ is not regular for $X$.

We set $\operatorname{Critic}(X)=\cup\{\hat{f} \mid f$ is critical for $X\}, \operatorname{Critic}(X)$ is the critical kernel of $X$. A face $f$ in $X$ is a maximal critical face, or an $M$-critical face for $X$, if $f$ is a principal face of $\operatorname{Critic}(X)$.

Again, the preceding definition of a regular cell is a generalization of the notion of a simple cell. As a corollary of Prop. 8, a principal face of a complex $X \preceq \mathbb{F}_{2}^{n}$ is regular for $X$ if and only if is simple for $X$.

We propose the following classification of critical faces which is specific to the $2 \mathrm{D}$ case. 


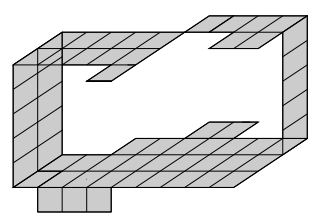

(a)

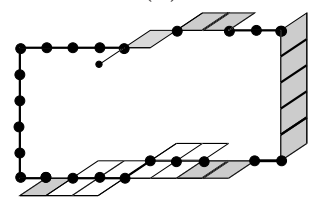

(c)

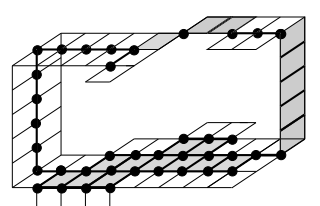

(b)

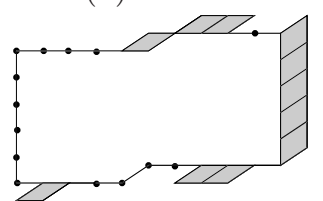

(d)

Fig. 2. (a): a complex $X_{0}$ in $\mathbb{F}_{2}^{3}$. (b): highlighted, $X_{1}=\operatorname{Critic}\left(X_{0}\right)$. (c): highlighted, $X_{2}=\operatorname{Critic}\left(X_{1}\right)$. (d): $X_{2}$ is such that $\operatorname{Critic}\left(X_{2}\right)=X_{2}$.

Definition 10. Let $X \preceq \mathbb{F}_{2}^{n}$, and let $f \in E s s(X)$.

i) $f$ is 0 -critical for $X$ if $\operatorname{Core}(\hat{f}, X)=\emptyset$;

ii) $f$ is 1-critical for $X$ if $\operatorname{Core}(\hat{f}, X)$ is not connected;

iii) $f$ is 2 -critical for $X$ if $f$ is an interior 2-face.

Note that a face $f$ is critical for $X \preceq \mathbb{F}_{2}^{n}$ if and only if $f$ is $k$-critical for some $k \in\{0,1,2\}$.

The following theorem holds for complexes of arbitrary dimensions (see [3]), it may be proved quite in a simple manner in the $2 \mathrm{D}$ case (first, we collapse regular 2 -faces onto their core, then we collapse regular 1-faces onto their core). This is our basic result in this framework. See Fig. 2 where the successive critical kernels of a complex are depicted.

Theorem 11. Let $X \preceq \mathbb{F}_{2}^{n}$. The critical kernel of $X$ is a retraction of $X$. Furthermore, if $Y \sqsubseteq X$ is such that $Y$ contains the critical kernel of $X$, then $Y$ is a retraction of $X$.

\section{Crucial kernels}

If $X$ is a complex in $\mathbb{F}_{2}^{n}$, the subcomplex $\operatorname{Critic}(X)$ is not necessarily a principal subcomplex of $X$ as illustrated Fig. 2. In this paper we investigate thinning algorithms which take as input a pure 2-complex and which return a principal subcomplex of the input (thus also a pure 2-complex). In this section, we propose some notions which allow to recover a principal subcomplex $Y$ of an arbitrary complex $X$, with the constraint that $Y$ is a retraction of $X$.

Definition 12. Let $X \preceq \mathbb{F}_{2}^{n}$, and let $f \in X^{+}$be a simple face for $X$.

We say that $f$ is crucial for $X$, if $\hat{f}^{*}$ contains a face which is M-critical for $X$. We say that $f$ is $k$-crucial for $X$, if $\hat{f}^{*}$ contains an M-critical face which is $k$-critical for $X, k=0,1$. 
(a)

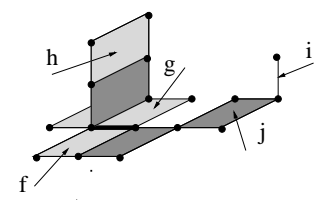

(b)

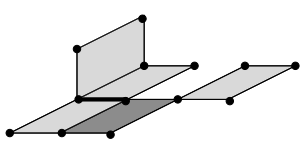

(c)

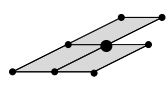

Fig. 3. (a): A complex $X_{0}$ and its M-critical faces (highlighted). (b): $X_{1}=\operatorname{Cruc}\left(X_{0}\right)$ and its M-critical faces. (c): The complex $X_{2}=\operatorname{Cruc}\left(X_{1}\right)$ contains only one M-critical face (highlighted), and $X_{2}=\operatorname{Cruc}\left(X_{2}\right)$.

Thus, a critical face for $X$ is either a principal face (which is not simple) or is included in a crucial face (which is also simple and principal).

In Fig. 3 (a), the M-critical faces of a complex are highlighted. The faces $f$ and $g$ are crucial (1-crucial), the faces $i$ and $h$ are simple but not crucial (the critical faces included in $i$ and $h$ are not M-critical), the face $j$ is not simple (it is M-critical), thus $j$ is not crucial.

Definition 13. Let $X \preceq \mathbb{F}_{2}^{n}$, and let $K$ be a set of crucial faces for $X$.

We say that $K$ is a $(k-)$ crucial clique for $X$, if there exists a ( $k$-critical) face $f$ which is M-critical for $X$ and such that $K$ is precisely the set of principal faces of $X$ which contain $f$. We also say that $K$ is the crucial clique induced by $f$.

In Fig. 3 (a), the set of faces $K=\{f, g\}$ is a 1-crucial clique, in (c) the set $K^{\prime}$ composed of the three 2 -faces is a 0 -crucial clique.

Definition 14. Let $X \preceq \mathbb{F}_{2}^{n}$ and let $Y \sqsubseteq X$.

We say that $Y$ is a crucial retraction of $X$ if:

i) $Y$ contains each principal face of $X$ which is critical; and

ii) $Y$ contains at least one face of each crucial clique for $X$.

From the above definitions, we immediately derive the following property.

Proposition 15. Let $X \preceq \mathbb{F}_{2}^{n}$ and let $Y \sqsubseteq X$.

We have Critic $(X) \subseteq Y$ if and only if $Y$ is a crucial retraction of $X$.

Thus, by Th. 11, if $Y$ is a crucial retraction of $X$, then $Y$ is a retraction of $X$. All algorithms proposed in this paper will iteratively compute crucial retractions.

Let us define the crucial kernel of $X$ as the set $\operatorname{Cruc}(X)$ which is the union of all cells of $X$ which are either not simple for $X$ or crucial for $X$. In Fig. 3 (a), a complex $X_{0}$ and its M-critical faces (three 2-faces and one 1-face) are depicted. The complex $X_{1}=\operatorname{Cruc}\left(X_{0}\right)$ is given in (b) also with its M-critical faces (one 2-face and one 1-face, which are both 1-critical). Finally, in (c), the complex $X_{2}=\operatorname{Cruc}\left(X_{1}\right)$ contains only one M-critical face (which is 0 -critical), and it may be seen that $X_{2}=\operatorname{Cruc}\left(X_{2}\right)$.

For thinning objects, we often want to keep other faces than the ones which are either not simple or crucial. That is why we introduce the following definition.

Definition 16. Let $X \preceq \mathbb{F}_{2}^{n}$. Let $P$ be a set of faces which are simple for $X$, and let $f \in P$. We say that $f$ is $(k-)$ crucial for $\langle X, P\rangle$, if $f$ belongs to a $(k-)$ crucial clique which is included in $P(k=0,1)$. 


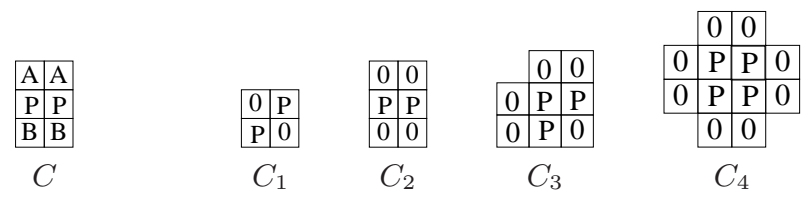

Fig. 4. Patterns and masks for crucial pixels. The 11 masks corresponding to these 5 patterns are obtained from them by applying any series of $\pi / 2$ rotations. The label 0 indicates pixels that must belong to the complement of $S$. The label $P$ indicates pixels that must belong to the set $P$ which is a set composed of simple pixels of $S$. For mask $C$, at least one of the pixels marked $\mathrm{A}$ and at least one of the pixels marked B must be in $S$. If one of these masks matches the sets $S, P$, then all the pixels which correspond to a label $P$ in the mask are recorded as "matched".

Intuitively, the set $P$ corresponds to a set of faces which are candidate for deletion in parallel. The following definition may be seen as a "template" for our thinning algorithms (see the expression of all the algorithms proposed in the next sections). Here, the set $K$ corresponds to a set which is preserved by a thinning algorithm (like extremities of curves, if we want to obtain a curvilinear skeleton).

Definition 17. Let $X \preceq \mathbb{F}_{2}^{n}$. Let $K$ be a set of principal faces of $X$, let $P$ be the set of faces in $X \backslash K$ which are simple for $X$, and let $R$ be the set composed of all faces which are crucial for $\langle X, P\rangle$. The set $\left[X^{+} \backslash P\right]^{-} \cup R^{-}$is the crucial kernel of $X$ constrained by $K$.

From the previous definitions, we immediately deduce the following proposition which ensures that any constrained crucial kernel preserves topology.

Proposition 18. Let $X \preceq \mathbb{F}_{2}^{n}$, and let $K$ be a set of principal faces of $X$. The crucial kernel of $X$ constrained by $K$ is a crucial retraction of $X$.

\section{Crucial pixels in the square grid}

We introduce the following definitions in order to establish a link between planar pure complexes (i.e., pure 2-complexes in $\mathbb{F}_{2}^{2}$ ) and the square grid as considered in image processing.

We define the square grid as the set $\mathbb{G}^{2}$ composed of all 2-faces of $\mathbb{F}_{2}^{2}$. A 2-face of $\mathbb{G}^{2}$ is also called a pixel. In the sequel, we consider only finite subsets of $\mathbb{G}^{2}$. For any pure 2-complex in $\mathbb{F}_{2}^{2}$, i.e., for any $X \sqsubseteq \mathbb{F}_{2}^{2}$, we associate the subset $X^{+}$of $\mathbb{G}^{2}$. In return, to each finite subset $S$ of $\mathbb{G}^{2}$, we associate the complex $S^{-}$of $\mathbb{F}_{2}^{2}$. This will be our basic methodology to "interpret" a set of pixels. In particular, all definitions given for a principal face in $X^{+}$have their counterparts for a pixel in $\mathbb{G}^{2}$. For example if $S \subseteq \mathbb{G}^{2}$ and $p \in S$, we will say that the pixel $p$ is simple for $S$ if $p$ is simple for $S^{-}$. Border, interior, $(k-)$ critical, and $(k-)$ crucial pixels are defined in the same manner. Observe that, if $p \in \mathbb{G}^{2}, \Gamma^{*}(p)$ and $\Gamma_{\mathcal{S}}^{*}(p)$ correspond to the so-called 8- and 4-neighborhood of $p$, respectively. We give now some simple local conditions, in the square grid, for crucial pixels. We express these local conditions by a set of masks, as in most papers related to 
parallel thinning in the digital topology framework. The definition of the masks $C, C_{1}, \ldots, C_{4}$ is given Fig. 4 .

Proposition 19. Let $S \subseteq \mathbb{G}^{2}, p \in S$, and let $P$ be a set of simple pixels of $S$.

i) The pixel $p$ is 1-crucial for $\langle S, P\rangle$ if and only if $p$ is matched by pattern $C$;

ii) The pixel $p$ is 0-crucial for $\langle S, P\rangle$ if and only if $p$ is matched by one of the patterns $C_{1}, \ldots C_{4}$.

Using the terminology of section 4 , the mask $C$ is a mask for 1-crucial cliques, and $C_{1}, \ldots, C_{4}$ are masks for 0-crucial cliques. For each of these masks, the crucial clique is the set composed of $P$ 's. In fact, it can be shown [5] that these masks also characterize the minimal non-simple sets introduced by C. Ronse [20], see also $[12,14]$. We observe that, since $P$ is composed of simple pixels of $S$, the set of $P$ 's of each mask $C_{1}, \ldots, C_{4}$ is necessarily surrounded by 0 's. Thus, we have:

Proposition 20. Let $S \subseteq \mathbb{G}^{2}$, and let $K$ be a 0-crucial clique for $S$. Then $K$ is a connected component of $S$.

\section{$6 \quad$ Minimal $\mathcal{K}$-skeletons}

A minimal symmetric skeleton of an object may be obtained by deleting iteratively, in parallel, all pixels which are neither critical nor crucial.

Definition 21. Let $S \subseteq \mathbb{G}^{2}$. The crucial kernel of $S$ is the set $\operatorname{Cruc}(S)$ which is composed of all critical pixels and all crucial pixels of $S$.

Let $\left\langle S_{0}, S_{1}, \ldots, S_{k}\right\rangle$ be the unique sequence such that $S_{0}=S, \operatorname{Cruc}\left(S_{k}\right)=S_{k}$ and $S_{i}=\operatorname{Cruc}\left(S_{i-1}\right), i=1, \ldots, k$. The set $S_{k}$ is the minimal $\mathcal{K}$-skeleton of $S$.

By Prop. 18 (here $K=\emptyset$ ), the minimal $\mathcal{K}$-skeleton of a set $S$ is a retraction of $S$. The following algorithm computes a minimal $\mathcal{K}$-skeleton. The pixels of $S$ which are kept at each step (04) of the algorithm correspond precisely to the pixels which are either critical (the set $S \backslash P$ ) or crucial (the set $R$ ).

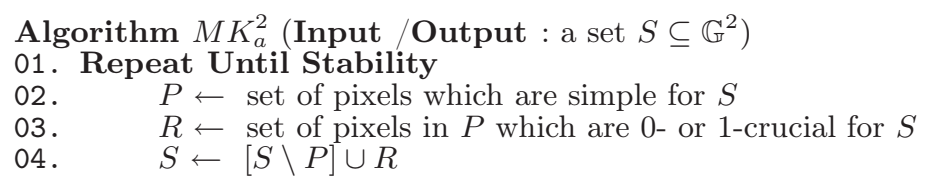

From Prop. 19, we may check if a pixel is 1-crucial by using the pattern $C$. Considering all possible rotations, there are in fact only two masks corresponding to $C$. On the other hand it may be seen that the checking of a 0 -crucial pixel with the patterns $C_{1}, \ldots, C_{4}$ involves 9 masks. In the following, we propose an algorithm which avoids the use of these 9 masks. This algorithm is based on a technic used for computing the so-called ultimate erosions in the context of mathematical morphology (see [24]).

Let $S \subseteq \mathbb{G}^{2}$, we denote by $S \ominus \Gamma^{*}=\left\{p \in S \mid \Gamma^{*}(p) \subseteq S\right\}$, the erosion of $S$ by $\Gamma^{*}$, and by $S \oplus \Gamma^{*}=\cup\left\{\Gamma^{*}(p) \mid p \in S\right\}$, the dilation of $S$ by $\Gamma^{*}$. 


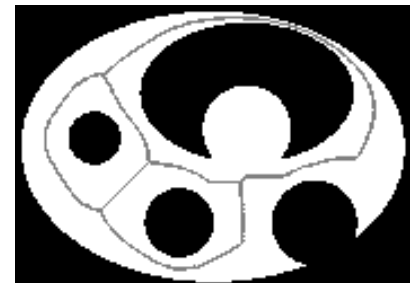

(a)

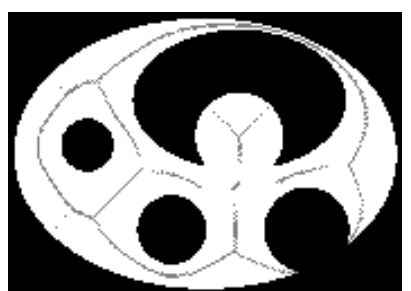

(b)

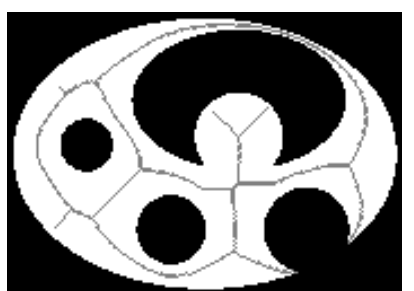

Fig. 5. (a): A subset $S$ of $\mathbb{G}^{2}$ (in white) and its minimal $\mathcal{K}$-skeleton (in gray). (b): The medial axis of $S$ (in gray). (c): in gray, $A K^{2}(S)$.

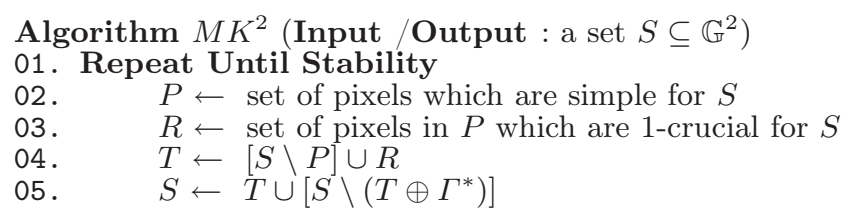

The correctness of the algorithm lies on the following property.

Proposition 22. Let $S \subseteq \mathbb{G}^{2}$, and let $p \in S$ be a simple pixel.

i) If $p$ is not crucial for $S$, then there exists $q \in \Gamma^{*}(p) \cap S$ such that $q$ is either critical or 1-crucial for $S$.

ii) If $p$ is 0-crucial for $S$, then any $q \in \Gamma^{*}(p) \cap S$ is neither critical, nor 1-crucial.

Let us denote by $M K^{2}(S)$ the result obtained by algorithm $M K^{2}$ from the input $S$. The set $T$ (line 04 ) is the set of pixels which are either critical or 1crucial. From Prop. 22, the pixels which are added to the set $T$ at step 05 of $M K^{2}$ are precisely 0 -crucial pixels. Thus, we have the following property.

Proposition 23. Let $S \subseteq \mathbb{G}^{2}$. The set $M K^{2}(S)$ is the minimal $\mathcal{K}$-skeleton of $S$.

An example of a minimal $\mathcal{K}$-skeleton is given Fig. 5 (a). As far as we know, $M K^{2}$ is the first algorithm for a minimal symmetric skeleton. Furthermore, the result of $M K^{2}$ is an object which is well-defined. To our best knowledge, this is also the first attempt to give a precise definition of such a notion.

\section{Topological axis and medial axis}

The quality of a curvilinear skeleton is often assessed by the fact that it contains, approximately or completely, the medial axis of the shape. We introduce the following definitions in order to generalize the medial axis for pure 2-complexes in $\mathbb{F}_{2}^{n}$, for arbitrary $n$.

Definition 24. Let $X \sqsubseteq \mathbb{F}_{2}^{n}$, and let $f \in X^{+}$. We set $\rho(f, X)$ as the minimum length of a collapse sequence of $X$ necessary to remove $f$ from $X$, if such a sequence exists, and $\rho(f, X)=\infty$ otherwise. We define the topological axis of $X$ as the set of faces $f$ in $X^{+}$such that $\rho(f, X)=\infty$ or $\rho(f, X) \geq \max \{\rho(g, X) \mid g \in$ $\Gamma_{\mathcal{S}}^{*}(f)$ and $\left.\rho(g, X) \neq \infty\right\}$. 
Note that we have $\rho(f, X)=1$ if and only if $f$ is a border face for $X$.

Let $X \sqsubseteq \mathbb{F}_{2}^{n}$, and let $f \in X^{+}$. We denote by $\pi^{\prime}(f, X)$ the length of a shortest strong path, in $X$, from $f$ to a border face of $X$, if such a path exists, and $\pi^{\prime}(f, X)=\infty$ otherwise. We denote by $\pi(f, X)$ the length of a shortest strong path, in $\mathbb{F}_{2}^{n}$, from $f$ to a border face of $X$. We observe that $\rho(f, X)=\pi^{\prime}(f, X)+1$. Now we focus our attention on the case $n=2$. Let $X \sqsubseteq \mathbb{F}_{2}^{2}$, and let $f \in X^{+}$. We have necessarily $\rho(f, X) \neq \infty$. Furthermore, since any 1 -face in $\mathbb{F}_{2}^{2}$ is included in precisely two 2-faces, it may be seen that $\pi(f, X)=\pi^{\prime}(f, X)$, thus $\rho(f, X)=$ $\pi(f, X)+1$.

In [22], A. Rosenfeld and J.L. Pfaltz have proved that, for the city-block and the chessboard distance, the medial axis of a shape can be obtained by detecting the local maxima of its distance transform, the medial axis being defined as the set of the centers of all the maximal balls for $S$. From the definition of the topological axis, and from the preceding remarks, we may deduce that the medial axis of $S$ with the city-block distance is precisely the topological axis of $S^{-}$. This shows that the notion of topological axis indeed generalizes the one of medial axis (which is not defined for the case of two dimensional structures in discrete $n$-dimensional spaces, $n>2$ ).

\section{$8 \mathcal{K}$-skeletons and medial axis}

For obtaining a skeleton which includes the medial axis of an object, we define the following notion of $\mathcal{K}$-skeleton which is constrained to include a given set $K$.

Definition 25. Let $S \subseteq \mathbb{G}^{2}$ and let $K \subseteq S$. Let $P$ be the set composed of all simple pixels for $S$ which are not in $K$. We denote by $\operatorname{Cruc}(S, K)$ the set composed of all pixels in $S \backslash P$ and all pixels which are crucial for $\langle S, P\rangle$.

Let $\left\langle S_{0}, S_{1}, \ldots, S_{k}\right\rangle$ be the unique sequence such that $S_{0}=S, S_{k}=\operatorname{Cruc}\left(S_{k}, K\right)$ and $S_{i}=\operatorname{Cruc}\left(S_{i-1}, K\right), i=1, \ldots, k$. The set $S_{k}$ is the $\mathcal{K}$-skeleton of $S$ constrained by $K$.

Again, by Prop 18, the $\mathcal{K}$-skeleton of a set $S$ constrained by a set $K$ is a retraction of $S$. We give now a general result on constrained thinning which permits, under some conditions, to avoid the checking of the 9 masks (corresponding to $\left.C_{1}, \ldots, C_{4}\right)$ for the detection of 0 -crucial pixels. This result is a direct consequence of Prop. 20.

Proposition 26. Let $S \subseteq \mathbb{G}^{2}$. Let $K \subseteq S$, such that each connected component of $S$ contains at least one pixel of $K$, and let $P$ be the set composed of all simple pixels for $S$ which are not in $K$. Then, any $p \in P$ is necessarily not 0 -crucial for $\langle S, P\rangle$.

For computing a $\mathcal{K}$-skeleton constrained by the medial axis, we could first extract the medial axis, and then compute the constrained skeleton, this method is followed by B.K. Jang and R.T. Chin [13]. We present here an algorithm which computes at the same time the medial axis and the skeleton. 


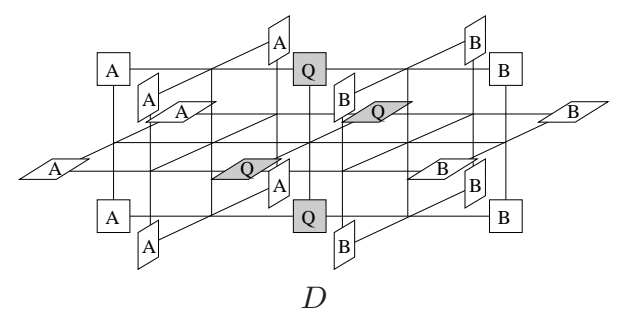

Fig. 6. Pattern and masks for 1-crucial surfels. The masks corresponding to this pattern are obtained by applying any series of $\pi / 2$ rotations. The label $Q$ indicates surfels that must either be in $P$ or in the complement of $S$; at least two surfels labeled $Q$ must be in $P$. At least one of the surfels marked $A$ and at least one of the surfels marked $B$ must be in $S$. If one of these masks matches the sets $S, P$, then all the surfels of $P$ which correspond to a label $Q$ are recorded as "matched".

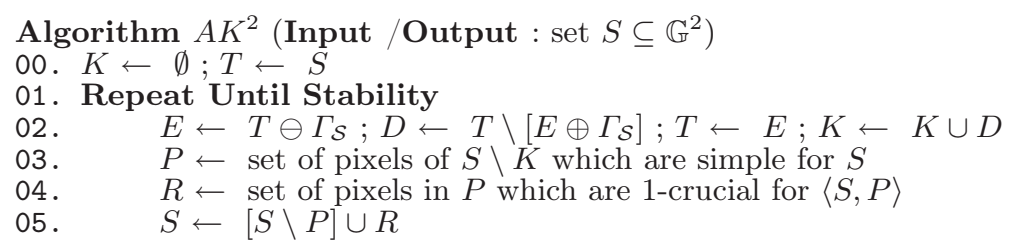

If we denote by $A K^{2}(S)$ the result obtained by algorithm $A K^{2}$, we have:

Proposition 27. Let $S \subseteq \mathbb{G}^{2}$. The set $A K^{2}(S)$ is the $\mathcal{K}$-skeleton of $S$ constrained by the topological axis of $S$.

In Fig. 5, we show a subset $S$ of $\mathbb{G}^{2}$ together with its topological (medial) axis (b) and its medial $\mathcal{K}$-skeleton (c). As far as we know, $A K^{2}$ is the first algorithm for a symmetric skeleton which contains the medial axis.

\section{$9 \mathcal{K}$-skeletons of $2 \mathrm{D}$ objects in $3 \mathrm{D}$ grids}

We consider in this section objects which are pure 2-complexes in $\mathbb{F}_{2}^{3}$. We denote by $\mathbb{G}_{2}^{3}$ the set composed of all 2 -faces of $\mathbb{F}_{2}^{3}$. A 2 -face of $\mathbb{G}_{2}^{3}$ is also called a surfel. In the sequel, we consider only finite subsets of $\mathbb{G}_{2}^{3}$.

As for the square grid, definitions of principal faces of $\mathbb{F}_{2}^{3}$ have their counterparts in $\mathbb{G}_{2}^{3}$. For example, if $S \subseteq \mathbb{G}_{2}^{3}$ and $p \in S$, we say that the surfel $p$ is simple for $S$ if $p$ is simple for $S^{-}$.

In the square grid, we were able to give a combinatorial characterization of 0and 1-crucial pixels. In fact, the number of configurations for 0-crucial surfels is too high for being directly exhibited. Fortunately, such a characterization is not mandatory to implement parallel thinning operators based on crucial kernels. It is possible to have a characterization for 1-crucial surfels which is based solely on the pattern $D$ given Fig. 6 .

Proposition 28. Let $S \subseteq \mathbb{G}_{2}^{3}, p \in S$. Let $P$ be a set of simple surfels of $S$. The surfel $p$ is 1-crucial for $\langle S, P\rangle$ if and only if $p$ is matched by the pattern $D$. 

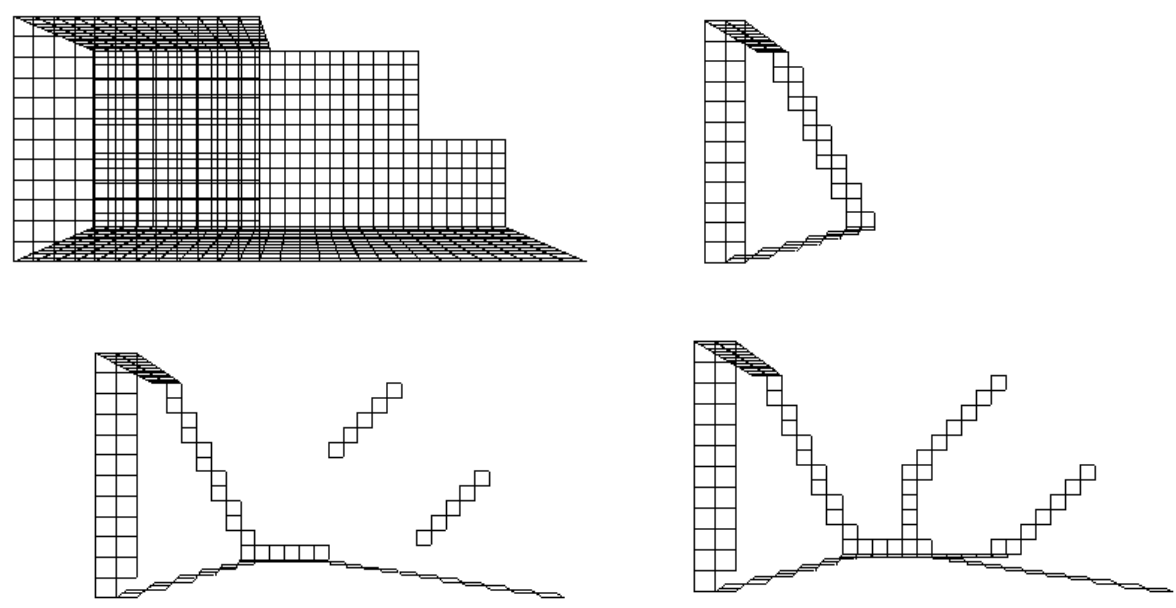

Fig. 7. Top left: A set of surfels $S$ in $\mathbb{F}_{2}^{3}$. Top right: The minimal $\mathcal{K}$-skeleton of $S$. Bottom left: The topological axis of $S$. Bottom right: The result of algorithm $B K_{2}^{3}$.

The following algorithm computes a minimal $\mathcal{K}$-skeleton, it has exactly the same structure as algorithm $M K^{2}$ for a square grid, but here, the checking of 1-crucial elements is made with the mask $D$.

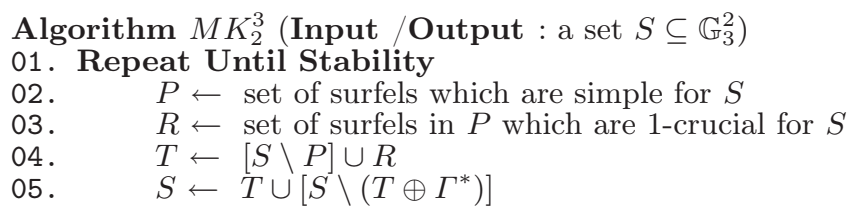

The topological soundness of the algorithm may be proved by establishing the analogue of Prop. 22 in $\mathbb{G}_{2}^{3}$. An example of a minimal $\mathcal{K}$-skeleton is given Fig. 7. As far as we know, $M K_{2}^{3}$ is the first algorithm for a minimal symmetric skeleton for an object made of surfels.

In a similar way, algorithm $A K^{2}$ may be transposed to design an algorithm which produces a skeleton containing the topological axis of an object which is made of surfels. We give here another example of an algorithm which has such a property.

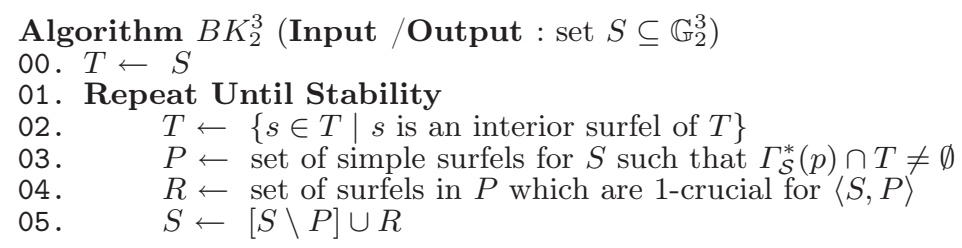


It may be seen that $B K_{2}^{3}(S)$, the result obtained by $B K_{2}^{3}$ from the input $S$, contains the topological axis of $S$. The topological soundness may be proved by establishing the equivalent of Prop. 26 in $\mathbb{G}_{2}^{3}$.

An example of a skeleton obtained with $B K_{2}^{3}$ is given Fig. 7 . To our best knowledge, there is only one other algorithm for symmetric curvilinear skeletons of $2 \mathrm{D}$ objects in 3D spaces which is the one given by J. Burguet and R. Malgouyres [6]. This algorithm is based on the technic of $\mathrm{P}$-simple points [2]. The $2 \mathrm{D}$ objects which are considered are the sets of surfels which constitute the boundary of $3 \mathrm{D}$ objects, or subsets of such boundaries. In this context, surfels which share a point or an interval are not necessarily considered as adjacent which makes a difference with the notion of adjacency used in this section. Another difference is that our algorithm always produce a skeleton which contains the topological axis of the original object.

\section{Conclusion}

Based on the framework of critical kernels [3], we studied the case of 2D structures in 2D and 3D grids. The salient outcomes of this article are the following:

- the definition and some characterizations of crucial faces, allowing for fast and simple implementations,

- the definition and an algorithm for a minimal symmetric skeleton $\left(M K^{2}\right)$,

- the introduction of the topological axis, which generalizes the medial axis,

- a parallel algorithm for a symmetric skeleton which contains the medial axis,

- a parallel algorithm for a minimal symmetric skeleton of an object made of surfels,

- a parallel algorithm for a symmetric skeleton, which contains the topological axis of an object made of surfels.

As far as we know, all the above algorithms have no equivalent.

In future works, we will study the case of general skeletons (i.e., which are not necessarily principal subcomplexes), and the important case of parallel thinning of $3 \mathrm{D}$ objects [4].

\section{References}

[1] C. Arcelli, L.P. Cordella, and S. Levialdi. Parallel thinning of binary pictures. Electronic Letters, 11(7):148-149, 1975.

[2] G. Bertrand. On P-simple points. Comptes Rendus de l'Académie des Sciences, Série Math., I(321):1077-1084, 1995.

[3] G. Bertrand. On critical kernels. Internal Report, Université de Marne-la-Vallée, IGM2005-05, 2005. Also submitted for publication.

[4] G. Bertrand and M. Couprie. Three-dimensional parallel thinning algorithms based on critical kernels. In preparation.

[5] G. Bertrand and M. Couprie. Two-dimensional parallel thinning algorithms based on critical kernels. Internal Report, Université de Marne-la-Vallée, IGM2006-02, 2006. Also submitted for publication. 
[6] J. Burguet and R. Malgouyres. Strong thinning and polyhedric approximation of the surface of a voxel object. Discrete Applied Mathematics, 125:93-114, 2003.

[7] R.T. Chin, H.K. Wan, D.L. Stover, and R.D. Iverson. A one-pass thinning algorithm and its parallel implementation. Computer Vision, Graphics, and Image Processing, 40(1):30-40, October 1987.

[8] M. Couprie. Note on fifteen 2d parallel thinning algorithms. Internal Report, Université de Marne-la-Vallée, IGM2006-01, 2005.

[9] U. Eckhardt and G. Maderlechner. Invariant thinning. International Journal of Pattern Recognition and Artificial Intelligence, 7(5):1115-1144, 1993.

[10] P. Giblin. Graphs, surfaces and homology. Chapman and Hall, 1981.

[11] R.W. Hall. Fast parallel thinning algorithms: Parallel speed and connectivity preservation. Communication of the ACM, 32(1):124-131, January 1989.

[12] R.W. Hall. Tests for connectivity preservation for parallel reduction operators. Topology and its Applications, 46(3):199-217, 1992.

[13] B.K. Jang and R.T. Chin. Reconstructable parallel thinning. Pattern Recognition and Artificial Intelligence, 7:1145-1181, 1993.

[14] T. Y. Kong. On topology preservation in 2-d and 3-d thinning. International Journal on Pattern Recognition and Artificial Intelligence, 9:813-844, 1995.

[15] T. Y. Kong. Topology-preserving deletion of 1's from 2-, 3- and 4-dimensional binary images. In Lecture Notes in Computer Science, volume 1347, pages 3-18, 1997.

[16] T. Y. Kong and A. Rosenfeld. Digital topology: introduction and survey. Comp. Vision, Graphics and Image Proc., 48:357-393, 1989.

[17] V.A. Kovalevsky. Finite topology as applied to image analysis. Computer Vision, Graphics and Image Processing, 46:141-161, 1989.

[18] A. Manzanera and T.M. Bernard. Metrical properties of a collection of $2 \mathrm{~d}$ parallel thinning algorithms. In Electronic Notes on Discrete Mathematics, Proc. 9th IWCIA, volume 12, 2003.

[19] T. Pavlidis. An asynchronous thinning algorithm. Computer Graphics and Image Processing, 20(2):133-157, October 1982.

[20] C. Ronse. Minimal test patterns for connectivity preservation in parallel thinning algorithms for binary digital images. Discrete Applied Mathematics, 21(1):67-79, 1988.

[21] A. Rosenfeld. Connectivity in digital pictures. Journal of the Association for Computer Machinery, 17:146-160, 1970.

[22] A. Rosenfeld and J.L. Pfaltz. Sequential operations in digital picture processing. Journal of the Association for Computer Machinery, 13:471-494, 1966.

[23] D. Rutovitz. Pattern recognition. Journal of the Royal Statistical Society, 129:504530, 1966.

[24] J. Serra. Image analysis and mathematical morphology. Academic Press, 1982.

[25] R. Stefanelli and A. Rosendeld. Some parallel thinning algorithms for digital pictures. Journal of the Association for Computing Machinery, 18(2):255-264, 1971. 